\title{
Structural Characterisation of Amorphous Solid Dispersions via Metropolis Matrix Factorisation of Pair Distribution Function Data
}

Harry Geddes, Helen Blade, James McCabe, Leslie P. Hughes, Andrew Goodwin

Submitted date: 20/08/2019 - Posted date: 21/08/2019

Licence: CC BY-NC-ND 4.0

Citation information: Geddes, Harry; Blade, Helen; McCabe, James; Hughes, Leslie P.; Goodwin, Andrew (2019): Structural Characterisation of Amorphous Solid Dispersions via Metropolis Matrix Factorisation of Pair Distribution Function Data. ChemRxiv. Preprint.

We measure the X-ray pair distribution functions (PDFs) of a series of felodipine:copovidone amorphous solid dispersions. Using a newly-developed Metropolis Matrix Factorisation (MMF) algorithm we extract from these data the PDF of the amorphous felodipine component in isolation. Our MMF analysis allows quantification of the degree of drug crystallinity in each sample, and structural characterisation of the amorphous drug viaits PDF. Comparison with atomistic simulations reveals that the (in)accessibility of conformational rotamers distinguishes amorphous and crystalline felodipine, in turn suggesting design routes for stabilising the amorphous form. We discuss the conceptual importance of our results in the context of characterising not only amorphous pharmaceuticals, but complex mixtures in general.

File list (2)

chemrxiv_2019_mmf.pdf (631.01 KiB) view on ChemRxiv • download file 


\title{
Structural Characterisation of Amorphous Solid Dispersions via Me- tropolis Matrix Factorisation of Pair Distribution Function Data
}

\author{
Harry S. Geddes, ${ }^{a}$ Helen Blade, ${ }^{\mathrm{b}}$ James F. McCabe, ${ }^{\mathrm{b}}$ Leslie P. Hughes, ${ }^{\mathrm{b}}$ and Andrew L. Goodwin ${ }^{\mathrm{a}}$

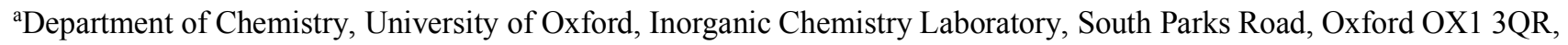 \\ U.K.

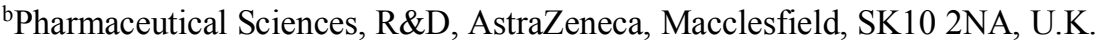

Supporting Information Placeholder

\begin{abstract}
We measure the X-ray pair distribution functions (PDFs) of a series of felodipine:copovidone amorphous solid dispersions. Using a newly-developed Metropolis Matrix Factorisation (MMF) algorithm we extract from these data the PDF of the amorphous felodipine component in isolation. Our MMF analysis allows quantification of the degree of drug crystallinity in each sample, and structural characterisation of the amorphous drug via its PDF. Comparison with atomistic simulations reveals that the (in)accessibility of conformational rotamers distinguishes amorphous and crystalline felodipine, in turn suggesting design routes for stabilising the amorphous form. We discuss the conceptual importance of our results in the context of characterising not only amorphous pharmaceuticals, but complex mixtures in general.
\end{abstract}

Amorphous solid dispersions (ASDs) are commonly used to improve the solubility - and hence bioavailability-of poorly water-soluble molecular pharmaceuticals. ${ }^{1-5}$ They comprise an amorphous active pharmaceutical ingredient (API) dispersed in a water-soluble polymer. An intrinsic limitation of such formulations is that they are thermodynamically unstable with respect to crystallisation. So the ability to monitor and optimise the structural integrity of ASDs is crucial because API crystallisation can impact severely on product performance. ${ }^{2}$ In this context, there are two key challenges for the structural chemist. The first is to devise experimental methodologies for characterising the API in its amorphous or partially-crystalline states within an ASD. The second is to establish what chemical and/or structural motifs help stabilise APIs in their amorphous form, ${ }^{6-8}$ so that one might predict formulation stability $a b$ initio rather than having to rely on lengthy real-time stability studies. In other words, what rules might we follow to make APIs amorphous by design?
Pair distribution function (PDF) methods are well suited to studying disordered systems such as ASDs. ${ }^{6,7}$ The PDF is an experimental measure of pairwise structural correlations, obtained as the Fourier transform of the $\mathrm{X}$-ray or neutron total scattering pattern - essentially a careful powder-diffraction measurement optimised for low backgrounds and maximum reciprocal-space coverage. Crucially, the PDF is well defined irrespective of the presence or absence of long-range order. Previous PDF studies of pharmaceuticals include structural investigations of crystalline APIs ${ }^{8,9}$ and fingerprinting of various amorphous and nanocrystalline drugs. ${ }^{10-12}$ Because there are no routine approaches for interpreting the PDFs of mixtures, nearly all studies have focussed on single-component APIs rather than on formulations (e.g. ASDs). ${ }^{12-19}$

In this study, we show how PDF measurements of ASDs with different API loadings allow extraction and quantification of the PDF for the amorphous API as if measured in isolation. We demonstrate this explicitly for ASDs prepared from felodipine $\left(\mathrm{a} \mathrm{Ca}^{2+}\right.$ antagonist used to treat hypertension) and copovidone (a water-soluble polymer binder), determining for the first time the PDF of amorphous felodipine, itself unobtainable in a pure form. Our analysis also quantifies the amount of amorphous and crystalline API present in each ASD, from which we calculate a 'crystallinity index'. Using this metric we establish a maximum stable API loading. Finally, we show that the experimental PDF of amorphous felodipine in its ASDs is remarkably well described by the PDF calculated from atomistic simulations of felodipine melt. We suggest that the (in)accessibility of conformational rotamers distinguishes the amorphous and crystalline states of felodipine, in turn suggesting design routes for stabilising its amorphous form.

At the heart of our study is a new hybrid multivariate analysis algorithm we term Metropolis Matrix Factorisation (MMF). Like all multivariate approaches - including the widely-used principal component analysis (PCA) method $^{20}-\mathrm{MMF}$ aims to describe a collection of $m$ 
experimental datasets (e.g. the ASD PDFs) in terms of weighted linear combinations of $n$ fundamental components $(n<m)$. Mathematically, there is no unique set of fundamental components - e.g. if combinations of $A$ and $B$ can describe our datasets, then so can those of $1 / 2(A+B)$ and $1 / 2(A-B)$. But there may be a unique set that is physically meaningful: in the present context, for example, the three fundamental components would be the PDFs of amorphous API, crystalline API, and polymer binder. PCA fails to find these because it forces components to be orthogonal; PDFs are probability functions, positive everywhere, and so are never orthogonal. Indeed the difficulty of interpreting PCA analyses of PDF measurements is well recognised in the field. ${ }^{21,22}$ Non-negative matrix factorisation (NMF) is a related approach which constrains components to be positive but does not require orthogonality. ${ }^{23-25}$ Whereas PCA is exact, NMF is only numerically soluble and as such is non-deterministic. The combination of random initialisation and least-squares minimisation used in its implementations means that it also often fails to find the correct fundamental components. Our new MMF approach circumvents this problem by employing Metropolis Monte Carlo minimisation, which in turn allows robust solution of the NMF problem.

As proof-of-concept, and to demonstrate how the method works, we consider first the application of MMF to a series of simple two-component mixtures of (crystalline) caffeine and (amorphous) povidone. We prepared 11 mixtures in total, corresponding to caffeine mass fractions of $0,10, \ldots, 100 \%$ (see SI). The corresponding X-ray total scattering patterns were measured using a PANalytical Empyrean X-ray diffractometer fitted with an Ag anode $\left(Q_{\max }=20 \AA^{-1}\right)$ and a GaliPIX3D detector. These data were normalised using GUDRUNX, ${ }^{26,27}$ and transformed to PDFs [Fig. 1(a)]; we use the normalisation referred to as $G^{\prime}(r)$ in Ref. 28, omitting the prime notation for ease. We then collated these data into an 11-component vector $\mathbf{G}^{\exp }(r)$. The task for MMF was to identify the two fundamental components $G_{i}^{*}(r) \quad(i=1,2)$ and weights $w_{i j}$ $(j=1, \ldots, 11)$ so as to minimise $\left|\mathbf{G}^{\text {calc }}(r)-\mathbf{G}^{\exp }(r)\right|^{2}$, where $G_{j}^{\text {calc }}(r)=\sum_{i=1}^{2} w_{i j} G_{i}^{*}(r)$ are the elements of $\mathbf{G}^{\text {calc }}(r)$. We applied the additional constraints that $G_{i}^{*}(r)$ is positive for all $i$ and $r$, and that $\sum_{i=1}^{2} w_{i j}=1$ for all $j$. The initial values of $G_{i}^{*}(r)$ and $w_{i j}$ were assigned randomly, subject to these constraints. Each minimisation round involved random variations to these parameters, followed by calculation of the change in goodness-of-fit $\Delta \mid \mathbf{G}^{\text {calc }}(r)$ $\left.\mathbf{G}^{\exp }(r)\right|^{2}$ and subsequent acceptance or rejection according to the Monte Carlo criterion. Such rounds were repeated with increasingly stringent acceptance criteria (i.e. simulated annealing) until convergence was achieved. An animation of the fitting process is provided as SI.

The fundamental component PDFs $G_{i}^{*}(r)$ so obtained are shown in Fig. 1(b); we find almost perfect agreement with the experimental PDFs of pure providone and pure caffeine. Likewise, the $w_{i j}$ are shown in Fig. 1(c); these describe the fractions of the two components in each sample and agree with the nominal compositions. Consequently, we can be confident that MMF extracts meaningful PDFs and meaningful phase fractions from the experimental PDFs of an ensemble of mixtures of varying composition.
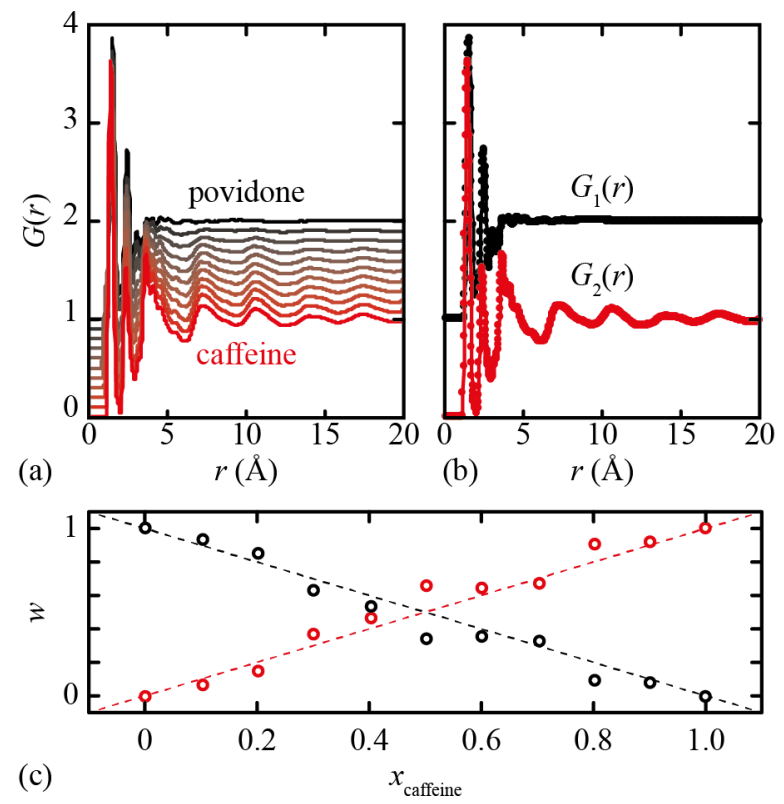

Figure 1. (a) X-ray PDFs for caffeine:povidone mixtures, with successive datasets shifted vertically by 0.1 units. Caffeine loadings range from $0 \%$ (black; top) to $100 \%$ (red; bottom). (b) MMF fundamental components (lines) compared to experimental $G(r)$ for povidone (black filled circles) and caffeine (red filled circles). (c) MMF coefficients $w$ as a function of caffeine loading. The dashed lines represent the ideal values.

Using the same experimental approach, we collected Xray PDF data for a family of felodipine:copovidone ASDs. The mass fractions of API in our series were $0 \%$, $15 \%, 20 \%, 30 \%$ and $50 \%$; samples were prepared by hot melt extrusion (see SI for details). Felodipine is not stabilisable in an amorphous form in the absence of polymer binder, ${ }^{29}$ so we also included in our series a sample of crystalline felodipine, giving a total of six samples. The corresponding X-ray PDF data are shown in Fig. 2(a).

MMF analysis of these data now involved deconvolution in terms of three fundamental components $G_{i}^{*}(r)$ $(i=1,2,3)$. We followed essentially the same approach as descried above, except that we fixed $G^{*}{ }_{1}(r)$ and $G_{2}^{*}(r)$ to be the PDFs of pure copovidone and crystalline felodipine, respectively, as we are no longer interested in proofof-concept but in determining most accurately the PDF of amorphous API. This PDF, as given by $G_{3}^{*}(r)$, is also shown in Fig. 2(a) $;^{30}$ the $w_{i j}$ are shown in Fig. 2(b).

Our results show clearly that for low felodipine loadings $(\leq 30 \%)$ the experimental PDF is well represented by a linear combination of the PDFs of copovidone binder and amorphous felodipine. Moreover, for the ASD with a felodipine loading of $50 \%$, a small but significant fraction 
of the crystalline felodipine PDF is needed to fit the experimental PDF. We suggest that the ratio of crystalline API to total (crystalline + amorphous) API components quantifies the degree of API crystallinity in a given dispersion. The value of this ratio, $\phi_{\text {cryst }}$, is shown for our six samples in the inset to Fig. 2(b). While other metrics have been proposed for quantifying crystallinity in ASDs, ${ }^{10,31}$ ${ }^{34}$ we believe this MMF ratio is the first to exploit PDF data. Quantification of the degree of crystallinity in ASDs - e.g. as a function of API loading, temperature, or humidity - may help identify maximum API loading, monitor API crystallisation, and play a role in quality control. In this particular case, our data suggest a mass fraction of $26 \%$ is the maximum API loading that can fully stabilise the amorphous form. This result is in quantitative agreement with previous dissolution experiments. ${ }^{35}$
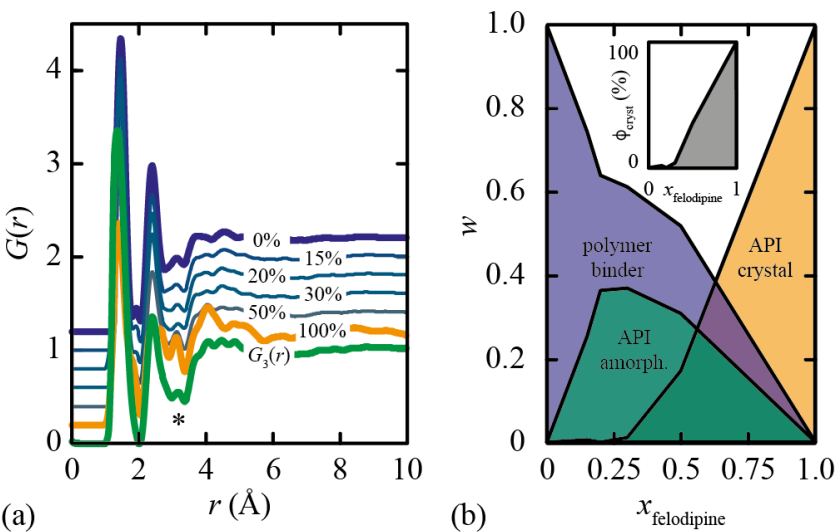

Figure 2. (a) X-ray PDFs of felodipine:copovidone ASDs with API loading indicated on each curve and successive curves shifted vertically by 0.2 units. The PDFs of pure (crystalline) API and pure copovidone (bold orange and indigo curves, respectively) were fixed as MMF components $G_{1}(r)$ and $G_{2}(r)$; the third component $G_{3}(r)$, which we interpret as the PDF of amorphous felodipine, was determined by MMF and is shown in green. The asterisk denotes a correlation at $r \sim 3 \AA$ that differs between crystalline and amorphous felodipine. (b) The corresponding MMF coefficients reveal the composition of each ASD. Inset: variation in API crystallinity index with loading.

But what of the previously unmeasurable PDF of amorphous felodipine? How sensible is it? And what might we learn regarding the mechanism of stabilisation of the amorphous state? In Fig. 2(a) we compare the PDFs of amorphous and crystalline felodipine. The two functions differ not only in the limiting behaviour at high- $r$, but also in the nature of correlations at distances $\sim 3 \AA$. This separation corresponds to intra-molecular atom-pairs, and so our data are consistent with a variation in felodipine conformation in ASDs relative to that adopted in the crystalline form. Intriguingly, molecular dynamics (MD) simulations of the felodipine melt (see SI for details) reflect precisely these same changes in PDF relative to that of the crystal: we compare the calculated and experimental functions in Fig. 3(a). The level of agreement is striking, and suggests that there is a close structural parallel between felodipine conformations sampled in its melt and amorphous states - despite the difference in temperature and the absence of polymer binder within the MD simulation. In Fig. 3(b) we show the distribution of molecular conformations reflected in a representative MD configuration. What is evident is the presence of ethoxy rotamers that are forbidden in the crystalline form. It is the alkoxy$\mathrm{Cl}$ pair correlations that occur at distances near $3 \AA$; these correlations are heavily weighted in the X-ray PDF as a consequence of the large electron density of $\mathrm{Cl}$. Consequently, we suggest that the progression from amorphous to crystalline felodipine involves population of a single alkoxy rotamer at the expense of others, presumably driven by crystal packing requirements. This hypothesis would account for the variation in PDFs at $r \sim 3 \AA$, and could be tested further using, for example, solid-state NMR measurements.
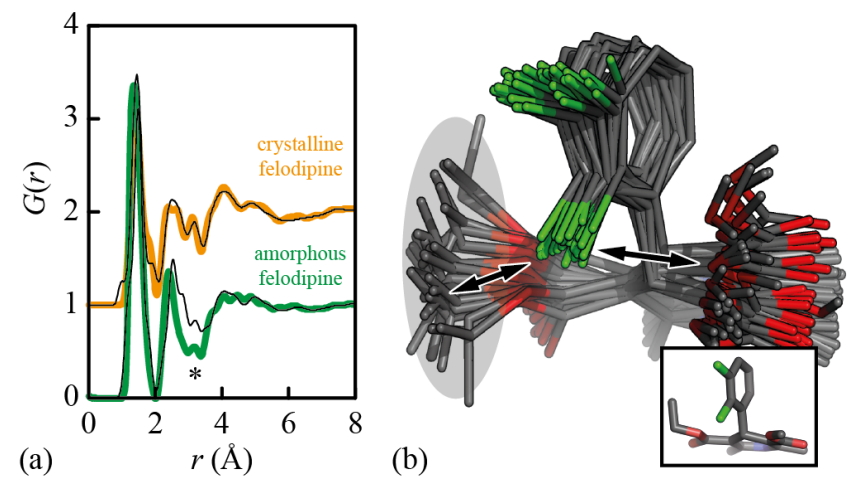

Figure 3. (a) X-ray PDFs of crystalline (orange curve, shifted vertically by 1 unit) and amorphous (green) felodipine, compared with PDFs calculated from the known crystal structure $^{36}$ and our MD simulations of felodipine melt (thin black curves; see SI for details). The different intensities of the feature at $r \sim 3 \AA$ in our experimental PDFs is reflected also in the calculated PDFs. (b) Superposition of molecular conformations in a representative $\mathrm{MD}$ configuration: $\mathrm{C}, \mathrm{O}$ and $\mathrm{Cl}$ atoms in grey, red, and green, respectively; $\mathrm{H}$ atoms omitted for clarity. The substantial conformational flexibility present-including different ethoxy rotamers (highlighted in grey) - is incompatible with the single conformation observed in the crystal (inset). The Cl-alkoxy separations (arrows) correspond to $r \sim 3 \AA$.

So on one level our results suggest possible design strategies for frustrating (re)crystallisation in the specific case of felodipine so as to stabilise the amorphous API at higher loadings; for example, increasing the energy barrier to alkoxy reorientation, incorporation of additional alkoxy substituents, and elongation and/or compositional variation of the alkoxy groups. But in arriving at these design rules, we have developed a new, general, and longneeded methodology for the structural characterisation and quantitative analysis of ASDs, offering clear advantages in quality control and drug design over existing approaches. What's more, this same methodology might be expected to transfer readily to the study of other 
complex mixtures beyond pharmaceuticals: e.g. battery materials, heterogeneous catalysts, and liquid fuels.

\section{ASSOCIATED CONTENT}

Supporting Information. The Supporting Information is available free of charge on the ACS Publications website.

\section{AUTHOR INFORMATION}

\section{Corresponding Author}

andrew.goodwin@chem.ox.ac.uk.

\section{Funding Sources}

No competing financial interests have been declared. The authors gratefully acknowledge financial support from the E.P.S.R.C. (studentship to H.S.G.) and the E.R.C. (Grants 279705 and 788144).

\section{ACKNOWLEDGMENT}

The authors gratefully acknowledge useful discussions with N. Funnell (ISIS).

\section{REFERENCES}

(1) Mureşan-Pop, M.; Pop, M. M.; Borodi, G.; Todea, M.; NagySimon, T.; Simon, S. Journal of Molecular Structure 2017, 1141, 607614.

(2) Vo, C. L.-N.; Park, C.; Lee, B.-J. Eur. J. Pharm. Biopharm. 2013, $85,799-813$.

(3) Song, Y.; Wang, L.; Yang, P.; Wenslow, R. M., Jr.; Tan, B.; Zhang, H.; Deng, Z. J. Pharm. Sci. 2013, 102, 1915-1923.

(4) Leuner, C.; Dressman, J. Eur. J. Pharm. Biopharm. 2000, 50, $47-60$.

(5) Chiou, W. L.; Reigelman, S. J. Pharm. Sci. 1969, 58, 1505-1510. 6476 .

(6) Young, C. A.; Goodwin, A. L. J. Mater. Chem. 2011, 21, 6464

(7) T. Egami and S. J. Billinge, Underneath the Bragg peaks: structural

analysis of complex materials, Pergamon, Oxford, 2003, vol. 7.

(8) Prill, D.; Juhas, P.; Schmidt, M. U.; Billinge, S. J. L. J. Appl. Cryst. 2015, 48 (1), 171-178. 3890 .

(9) Chen, S.; Sheikh, A. Y.; Ho, R. J. Pharm. Sci. 2014, 103, 3879-

(10) Benmore, C. J.; Weber, J. K. R.; Tailor, A. N.; Cherry, B. R.; Yarger, J. L.; Mou, Q.; Weber, W.; Neuefeind, J.; Byrn, S. R. J. Pharm. Sci. 2013, 102, 1290-1300.

(11) Dykhne, T.; Taylor, R.; Florence, A.; Billinge, S. J. L. Pharm. Res. 2011, 28, 1041-1048.

(12) Billinge, S. J. L.; Dykhne, T.; Juhás, P.; Božin, E.; Taylor, R.; Florence, A. J.; Shankland, K. CrystEngComm 2010, 12, 1366-1368.
(13) Bates, S.; Zografi, G.; Engers, D.; Morris, K.; Crowley, K.; Newman, A. Pharm. Res. 2006, 23, 2333-2349.

(14) Bates, S.; Kelly, R. C.; Ivanisevic, I.; Schields, P.; Zografi, G.; Newman, A. W. J. Pharm. Sci. 2007, 96, 1418-1433.

(15) Bøtker, J. P.; Karmwar, P.; Strachan, C. J.; Cornett, C.; Tian, F.; Zujovic, Z.; Rantanen, J.; Rades, T. Int. J. Pharm. 2011, 417, 112119.

(16) Naelapää, K.; Boetker, J. P.; Veski, P.; Rantanen, J.; Rades, T.; Kogermann, K. Int. J. Pharm. 2012, 429, 69-77.

(17) Davis, T.; Johnson, M.; Billinge, S. J. L. Cryst. Growth Des. 2013, 13, 4239-4244.

(18) Bordet, P.; Bytchkov, A.; Descamps, M.; Dudognon, E.; Elkaïm, E.; Martinetto, P.; Pagnoux, W.; Poulain, A.; Willart, J.-F. Cryst. Growth Des. 2016, 16, 4547-4558.

(19) Terban, M. W.; Cheung, E. Y.; Krolikowski, P.; Billinge, S. J. L. Cryst. Growth Des. 2016, 16, 210-220.

(20) Jolliffe, I. T. 2002 Principal component analysis, 2nd edn. New York, NY: Springer-Verlag.

(21) Wang, Y.-X.; Zhang, Y.-J. IEEE Trans. Knowl. Data Eng. 2013, 25, 1336-1353.

(22) Chapman, K. W.; Lapidus, S. H.; Chupas, P. J. J. Appl. Cryst. 2015, 48, 1619-1626.

(23) Seung, H. S.; Lee, D. D. Nature 1999, 401, 788-791.

(24) Lee, D. D.; Seung, H. S. Adv. Neural Inf. Process Syst. 2001, $13,556-562$.

(25) Berry, M. W.; Browne, M.; Langville, A. N.; Pauca, V. P.; Plemmons, R. J. Comput. Stat. Data Anal. 2007, 52, 155-173.

(26) Soper, A. K. GudrunN and GudrunX: Programs for Correcting Raw Neutron and X-ray Diffraction Data to Differential Scattering Cross Section. Tech. Rep. RAL-TR-2011-013 (Rutherford Appleton Laboratory, 2011).

(27) Soper, A. K.; Barney, J. Appl. Cryst. 2011, 44, 714-726.

(28) Keen, D. A.; J. Appl. Cryst. 2001, 34, 172-177.

(29) Marsac, P. J.; Konno, H.; Taylor, L. S. Pharm. Res. 2006, 23, 2306-2316

(30) Strictly, $G_{3}^{*}(r)$ will comprise the PDF of amorphous felodipine together with contributions from interfaces between different phases (e.g. API/binder). We make use of the same approximation exploited in difference PDF measurements ${ }^{22,37}$ that inter-phase PDFs are substantially less structured than intra-phase PDFs and hence $G_{3}^{*}(r)$ will be dominated by the contribution from amorphous API.

(31) Zidan, A. S.; Rahman, Z.; Sayeed, V.; Raw, A.; Yu, L.; Khan, M. A. Int. J. Pharm. 2012, 423, 341-350.

(32) Rumondor, A. C. F.; Taylor, L. S. Int. J. Pharm. 2010, 398, $155-160$

(33) Vo, C. L.-N.; Park, C.; Lee, B.-J. Eur. J. Pharm. Biopharm. 2013, 85, 799-813. 421 .

(34) Baird, J. A.; Taylor, L. S. Adv. Drug Deliv. Rev. 2012, 64, 396-

(35) Langham, Z. A.; Booth, J.; Hughes, L. P.; Reynolds, G. K.; Wren, S. A. C. J. Pharm. Sci. 2012, 101, 2798-2810.

(36) Fossheim, R. J. Med. Chem. 1986, 29, 305-307.

(37) Chupas, P. J.; Chapman, K. W.; Jennings, G.; Lee, P. L.; Grey, C. P. J. Am. Chem. Soc. 2007, 129, 13822-13824. 


\title{
Structural Characterisation of Amorphous Solid Dispersions via Metropolis Matrix Factorisation of Pair Distribution Function Data
}

\author{
Harry S. Geddes, ${ }^{a}$ Helen Blade, ${ }^{b}$ James F. McCabe, ${ }^{b}$ Leslie P. Hughes, ${ }^{b}$ and Andrew L. \\ Goodwin ${ }^{* *}$ \\ aDepartment of Chemistry, University of Oxford, Inorganic Chemistry Laboratory, South Parks Road, \\ Oxford OX1 3QR, U.K.

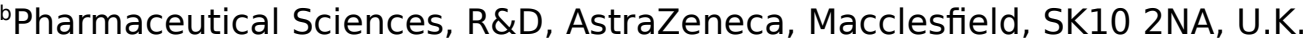

Supporting Information Placeholder

\begin{abstract}
We measure the X-ray pair distribution functions (PDFs) of a series of felodipine:copovidone amorphous solid dispersions. Using a newly-developed Metropolis Matrix Factorisation (MMF) algorithm we extract from these data the PDF of the amorphous felodipine component in isolation. Our MMF analysis allows quantification of the degree of drug crystallinity in each sample, and structural characterisation of the amorphous drug via its PDF. Comparison with atomistic simulations reveals that the (in)accessibility of conformational rotamers distinguishes amorphous and crystalline felodipine, in turn suggesting design routes for stabilising the amorphous form. We discuss the conceptual importance of our results in the context of characterising not only amorphous pharmaceuticals, but complex mixtures in general.
\end{abstract}

Amorphous solid dispersions (ASDs) are commonly used to improve the solubilityand hence bioavailability-of poorly watersoluble molecular pharmaceuticals. ${ }^{1-5}$ They comprise an amorphous active pharmaceutical ingredient (API) dispersed in a water-soluble polymer. An intrinsic limitation of such formulations is that they are thermodynamically unstable with respect to crystallisation. So the ability to monitor and optimise the structural integrity of ASDs is crucial because API crystallisation can impact severely on product performance. ${ }^{2}$ In this context, there are two key challenges for the structural chemist. The first is to devise experimental methodologies for characterising the API in its amorphous or partially-crystalline states within an ASD. The second is to establish what chemical and/or structural motifs help stabilise APIs in their amorphous form, ${ }^{6-8}$ so that one might predict formulation stability $a b$ initio rather than having to rely on lengthy real-time stability studies. In other words, what rules might we follow to make APIs amorphous by design?

Pair distribution function (PDF) methods are well suited to studying disordered systems such as ASDs. ${ }^{6,7}$ The PDF is an experimental measure of pairwise structural correlations, obtained as the Fourier transform of the Xray or neutron total scattering patternessentially a careful powder-diffraction measurement optimised for low backgrounds and maximum reciprocal-space coverage. Crucially, the PDF is well defined irrespective of the presence or absence of long-range order. Previous PDF studies of pharmaceuticals include structural investigations of crystalline $\mathrm{APIs}^{8,9}$ and fingerprinting of various amorphous and nanocrystalline drugs. ${ }^{10-12}$ Because there are no routine approaches for interpreting the PDFs of mixtures, nearly all studies have focussed on single-component APIs rather than on formulations (e.g. ASDs). ${ }^{12-19}$ 
In this study, we show how PDF measurements of ASDs with different API loadings allow extraction and quantification of the PDF for the amorphous API as if measured in isolation. We demonstrate this explicitly for ASDs prepared from felodipine $\left(a \mathrm{Ca}^{2+}\right.$ antagonist used to treat hypertension) and copovidone (a water-soluble polymer binder), determining for the first time the PDF of amorphous felodipine, itself unobtainable in a pure form. Our analysis also quantifies the amount of amorphous and crystalline API present in each ASD, from which we calculate a 'crystallinity index'. Using this metric we establish a maximum stable API loading. Finally, we show that the experimental PDF of amorphous felodipine in its ASDs is remarkably well described by the PDF calculated from atomistic simulations of felodipine melt. We suggest that the (in)accessibility of conformational rotamers distinguishes the amorphous and crystalline states of felodipine, in turn suggesting design routes for stabilising its amorphous form.

At the heart of our study is a new hybrid multivariate analysis algorithm we term Metropolis Matrix Factorisation (MMF). Like all multivariate approaches-including the widely-used principal component analysis (PCA) method ${ }^{20}$-MMF aims to describe a collection of $m$ experimental datasets (e.g. the ASD PDFs) in terms of weighted linear combinations of $n$ fundamental components $(n<m)$. Mathematically, there is no unique set of fundamental components-e.g. if combinations of $A$ and $B$ can describe our datasets, then so can those of $1 / 2(A+B)$ and $1 / 2(A-B)$. But there may be a unique set that is physically meaningful: in the present context, for example, the three fundamental components would be the PDFs of amorphous API, crystalline API, and polymer binder. PCA fails to find these because it forces components to be orthogonal; PDFs are probability functions, positive everywhere, and so are never orthogonal. Indeed the difficulty of interpreting PCA analyses of PDF measurements is well recognised in the field. ${ }^{21,22}$ Non-negative matrix factorisation (NMF) is a related approach which constrains components to be positive but does not require orthogonality. ${ }^{23-25}$ Whereas
PCA is exact, NMF is only numerically soluble and as such is non-deterministic. The combination of random initialisation and least-squares minimisation used in its implementations means that it also often fails to find the correct fundamental components. Our new MMF approach circumvents this problem by employing Metropolis Monte Carlo minimisation, which in turn allows robust solution of the NMF problem.

As proof-of-concept, and to demonstrate how the method works, we consider first the application of MMF to a series of simple two-component mixtures of (crystalline) caffeine and (amorphous) povidone. We prepared 11 mixtures in total, corresponding to caffeine mass fractions of $0,10, \ldots, 100 \%$ (see SI). The corresponding $\mathrm{X}$-ray total scattering patterns were measured using a PANalytical Empyrean Xray diffractometer fitted with an $\mathrm{Ag}$ anode $\left(Q_{\max }=20 \AA^{-1}\right)$ and a GaliPIX3D detector. These data were normalised using GUDRUNX, ${ }^{26,27}$ and transformed to PDFs [Fig. 1(a)]; we use the normalisation referred to as $G^{\prime}(r)$ in Ref. 28, omitting the prime notation for ease. We then collated these data into an 11-component vector $\mathbf{G}^{\exp }(r)$. The task for MMF was to identify the two fundamental components $G_{i}^{*}(r)$ $(i=1,2)$ and weights $w_{i j}(j=1, \ldots, 11)$ so as to minimise $\quad\left|\mathbf{G}^{\text {calc }}(r)-\mathbf{G}^{\exp }(r)\right|^{2}$, where $G_{j}^{\text {calc }}(r)=\sum_{i=1}^{2} w_{i j} G_{i}^{i}(r)$ are the elements of $\mathbf{G}^{\text {calc }}(r)$. We applied the additional constraints that $G_{i}^{*}(r)$ is positive for all $i$ and $r$, and that $\sum_{i=1}^{2} w_{i j}=1$ for all $j$. The initial values of $G_{i}^{*}(r)$ and $w_{i j}$ were assigned randomly, subject to these constraints. Each minimisation round involved random variations to these parameters, followed by calculation of the change in goodnessof-fit $\Delta\left|\mathbf{G}^{\text {calc }}(r)-\mathbf{G}^{\exp }(r)\right|^{2}$ and subsequent acceptance or rejection according to the Monte Carlo criterion. Such rounds were repeated with increasingly stringent acceptance criteria (i.e. simulated annealing) until convergence was achieved. An animation of the fitting process is provided as SI.

The fundamental component PDFs $G_{i}^{*}(r)$ so obtained are shown in Fig. 1(b); we find almost perfect agreement with the experimental PDFs of pure providone and 
pure caffeine. Likewise, the $w_{i j}$ are shown in Fig. 1(c); these describe the fractions of the two components in each sample and agree with the nominal compositions. Consequently, we can be confident that MMF extracts meaningful PDFs and meaningful phase fractions from the experimental PDFs of an ensemble of mixtures of varying composition.
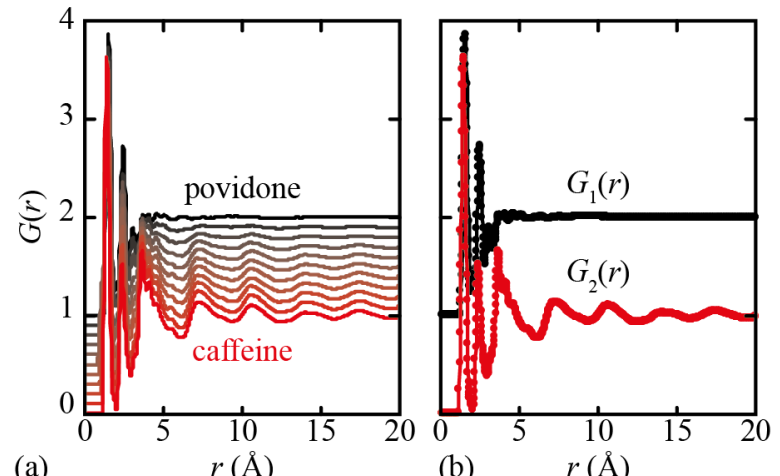

(a)

$r(\AA)$

(b) $\quad r(\AA)$

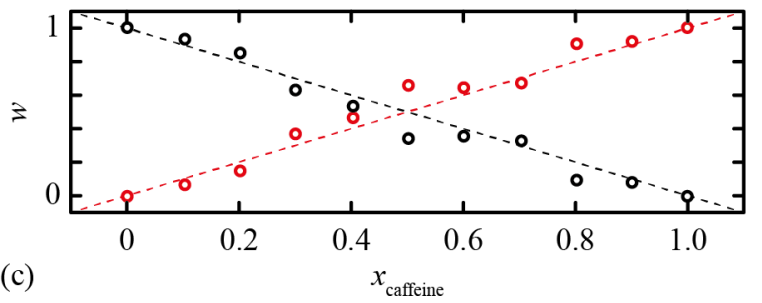

Figure 1. (a) X-ray PDFs for caffeine:povidone mixtures, with successive datasets shifted vertically by 0.1 units. Caffeine loadings range from $0 \%$ (black; top) to $100 \%$ (red; bottom). (b) MMF fundamental components (lines) compared to experimental $G(r)$ for povidone (black filled circles) and caffeine (red filled circles). (c) MMF coefficients $w$ as a function of caffeine loading. The dashed lines represent the ideal values.

Using the same experimental approach, we collected X-ray PDF data for a family of felodipine:copovidone ASDs. The mass fractions of API in our series were $0 \%$, $15 \%, 20 \%, 30 \%$ and $50 \%$; samples were prepared by hot melt extrusion (see SI for details). Felodipine is not stabilisable in an amorphous form in the absence of polymer binder, ${ }^{29}$ so we also included in our series a sample of crystalline felodipine, giving a total of six samples. The corresponding Xray PDF data are shown in Fig. 2(a).

MMF analysis of these data now involved deconvolution in terms of three fundamental components $G_{i}^{*}(r) \quad(i=1,2,3)$. We followed essentially the same approach as descried above, except that we fixed $G_{1}^{*}(r)$ and $G_{2}^{*}(r)$ to be the PDFs of pure copovidone and crystalline felodipine, respectively, as we are no longer interested in proof-of-concept but in determining most accurately the PDF of amorphous API. This PDF, as given by $G^{*}{ }_{3}(r)$, is also shown in Fig. $2(a) ;{ }^{30}$ the $w_{i j}$ are shown in Fig. 2(b).

Our results show clearly that for low felodipine loadings ( $\leq 30 \%$ ) the experimental PDF is well represented by a linear combination of the PDFs of copovidone binder and amorphous felodipine. Moreover, for the ASD with a felodipine loading of $50 \%$, a small but significant fraction of the crystalline felodipine PDF is needed to fit the experimental PDF. We suggest that the ratio of crystalline API to total (crystalline + amorphous) API components quantifies the degree of API crystallinity in a given dispersion. The value of this ratio, $\phi_{\text {cryst, }}$ is shown for our six samples in the inset to Fig. 2(b). While other metrics have been proposed for quantifying crystallinity in ASDs, ${ }^{10,31-34}$ we believe this MMF ratio is the first to exploit PDF data. Quantification of the degree of crystallinity in ASDs-e.g. as a function of API loading, temperature, or humidity - may help identify maximum API loading, monitor API crystallisation, and play a role in quality control. In this particular case, our data suggest a mass fraction of $26 \%$ is the maximum API loading that can fully stabilise the amorphous form. This result is in quantitative agreement with previous dissolution experiments. ${ }^{35}$
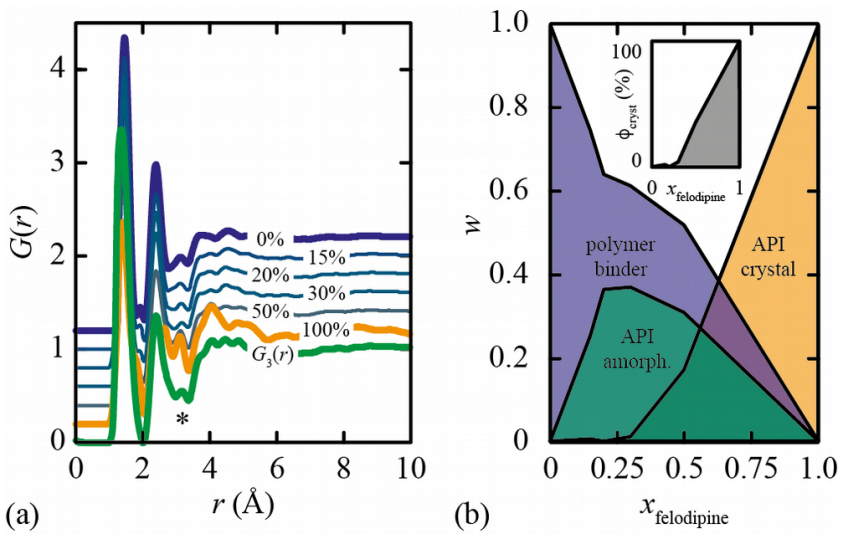

Figure 2. (a) X-ray PDFs of felodipine:copovidone ASDs with API loading indicated on each curve and successive curves shifted vertically by 0.2 units. The PDFs of pure (crystalline) API and pure copovidone (bold orange and indigo curves, respectively) were fixed as MMF components $G_{1}(r)$ and $G_{2}(r)$; the third component $G_{3}(r)$, which we interpret as the PDF of amorphous 
felodipine, was determined by MMF and is shown in green. The asterisk denotes a correlation at $r \sim 3 \AA$ that differs between crystalline and amorphous felodipine. (b) The corresponding MMF coefficients reveal the composition of each ASD. Inset: variation in API crystallinity index with loading.

But what of the previously unmeasurable PDF of amorphous felodipine? How sensible is it? And what might we learn regarding the mechanism of stabilisation of the amorphous state? In Fig. 2(a) we compare the PDFs of amorphous and crystalline felodipine. The two functions differ not only in the limiting behaviour at high-r, but also in the nature of correlations at distances $\sim 3 \AA$. This separation corresponds to intra-molecular atom-pairs, and so our data are consistent with a variation in felodipine conformation in ASDs relative to that adopted in the crystalline form. Intriguingly, molecular dynamics (MD) simulations of the felodipine melt (see SI for details) reflect precisely these same changes in PDF relative to that of the crystal: we compare the calculated and experimental functions in Fig. 3(a). The level of agreement is striking, and suggests that there is a close structural parallel between felodipine conformations sampled in its melt and amorphous states-despite the difference in temperature and the absence of polymer binder within the MD simulation. In Fig. 3(b) we show the distribution of molecular conformations reflected in a representative MD configuration. What is evident is the presence of ethoxy rotamers that are forbidden in the crystalline form. It is the alkoxy-Cl pair correlations that occur at distances near $3 \AA$; these correlations are heavily weighted in the X-ray PDF as a consequence of the large electron density of $\mathrm{Cl}$. Consequently, we suggest that the progression from amorphous to crystalline felodipine involves population of a single alkoxy rotamer at the expense of others, presumably driven by crystal packing requirements. This hypothesis would account for the variation in PDFs at $r \sim 3 \AA$, and could be tested further using, for example, solid-state NMR measurements.
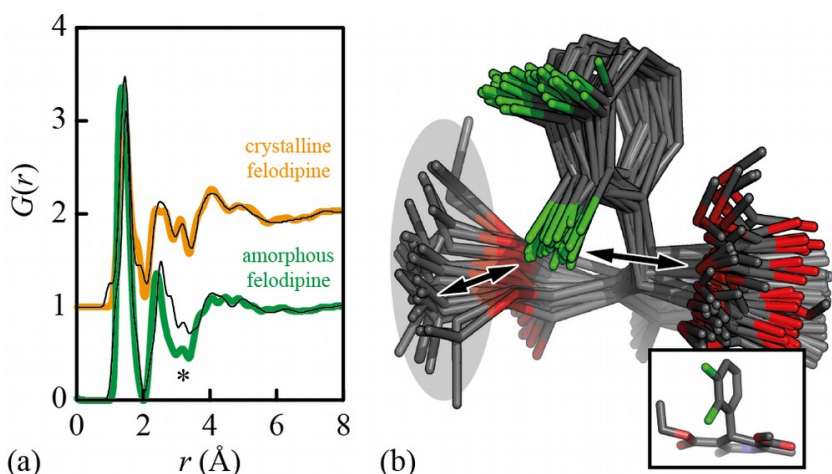

Figure 3. (a) X-ray PDFs of crystalline (orange curve, shifted vertically by 1 unit) and amorphous (green) felodipine, compared with PDFs calculated from the known crystal structure $^{36}$ and our MD simulations of felodipine melt (thin black curves; see SI for details). The different intensities of the feature at $r \sim 3 \AA$ in our experimental PDFs is reflected also in the calculated PDFs. (b) Superposition of molecular conformations in a representative MD configuration: $\mathrm{C}, \mathrm{O}$ and $\mathrm{Cl}$ atoms in grey, red, and green, respectively; $\mathrm{H}$ atoms omitted for clarity. The substantial conformational flexibility present -including different ethoxy rotamers (highlighted in grey)-is incompatible with the single conformation observed in the crystal (inset). The $\mathrm{Cl}$-alkoxy separations (arrows) correspond to $r \sim 3 \AA$.

So on one level our results suggest possible design strategies for frustrating (re)crystallisation in the specific case of felodipine so as to stabilise the amorphous API at higher loadings; for example, increasing the energy barrier to alkoxy reorientation, incorporation of additional alkoxy substituents, and elongation and/or compositional variation of the alkoxy groups. But in arriving at these design rules, we have developed a new, general, and long-needed methodology for the structural characterisation and quantitative analysis of ASDs, offering clear advantages in quality control and drug design over existing approaches. What's more, this same methodology might be expected to transfer readily to the study of other complex mixtures beyond pharmaceuticals: e.g. battery materials, heterogeneous catalysts, and liquid fuels.

\section{ASSOCIATED CONTENT}

Supporting Information. The Supporting Information is available free of charge on the ACS Publications website. 


\section{AUTHOR INFORMATION}

\section{Corresponding Author}

andrew.goodwin@chem.ox.ac.uk.

\section{Funding Sources}

No competing financial interests have been declared.

The authors gratefully acknowledge financial support from the E.P.S.R.C. (studentship to H.S.G.) and the E.R.C. (Grants 279705 and 788144).

\section{ACKNOWLEDGMENT}

The authors gratefully acknowledge useful discussions with N. Funnell (ISIS).

\section{REFERENCES}

(1) Mureşan-Pop, M.; Pop, M. M.; Borodi, G.; Todea, M.; Nagy-Simon, T.; Simon, S. Journal of Molecular Structure 2017, 1141, 607-614.

(2) Vo, C. L.-N.; Park, C.; Lee, B.-J. Eur. J. Pharm. Biopharm. 2013, 85, 799-813.

(3) Song, Y.; Wang, L.; Yang, P.; Wenslow, R. M., Jr.; Tan, B.; Zhang, H.; Deng, Z. J. Pharm. Sci. 2013, 102, 1915-1923.

(4) Leuner, C.; Dressman, J. Eur. J. Pharm. Biopharm. 2000, 50, 47-60.

(5) Chiou, W. L.; Reigelman, S. J. Pharm. Sci. 1969, 58, 1505-1510.

(6) Young, C. A.; Goodwin, A. L. J. Mater. Chem. 2011, 21, 6464-6476.

(7) T. Egami and S. J. Billinge, Underneath the Bragg peaks: structural

analysis of complex materials, Pergamon, Oxford, 2003, vol. 7.

(8) Prill, D.; Juhas, P.; Schmidt, M. U.; Billinge, S. J. L. J. Appl. Cryst. 2015, 48 (1), 171-178.

(9) Chen, S.; Sheikh, A. Y.; Ho, R. J. Pharm. Sci. 2014, 103, 3879-3890.

(10) Benmore, C. J.; Weber, J. K. R.; Tailor, A. N.; Cherry, B. R.; Yarger, J. L.; Mou, Q.; Weber, W.; Neuefeind, J.; Byrn, S. R. J. Pharm. Sci. 2013, 102, 1290-1300.

(11) Dykhne, T.; Taylor, R.; Florence, A.; Billinge, S. J. L. Pharm. Res. 2011, 28, 1041-1048.

(12) Billinge, S. J. L.; Dykhne, T.; Juhás, P.; Božin, E.; Taylor, R.; Florence, A. J.; Shankland, K. CrystEngComm 2010, 12, 1366-1368.

(13) Bates, S.; Zografi, G.; Engers, D.; Morris, K.; Crowley, K.; Newman, A. Pharm. Res. 2006, 23, 2333-2349.

(14) Bates, S.; Kelly, R. C.; Ivanisevic, I.; Schields, P.; Zografi, G.; Newman, A. W. J. Pharm. Sci. 2007, 96, 1418-1433.

(15) Bøtker, J. P.; Karmwar, P.; Strachan, C. J.; Cornett, C.; Tian, F.; Zujovic, Z.; Rantanen, J.; Rades, T. Int. J. Pharm. 2011, 417, 112-119.
(16) Naelapää, K.; Boetker, J. P.; Veski, P.; Rantanen, J.; Rades, T.; Kogermann, K. Int. J. Pharm. 2012, 429, 69-77.

(17) Davis, T.; Johnson, M.; Billinge, S. J. L. Cryst. Growth Des. 2013, 13, 4239-4244.

(18) Bordet, P.; Bytchkov, A.; Descamps, M.; Dudognon, E.; Elkaïm, E.; Martinetto, P.; Pagnoux, W.; Poulain, A.; Willart, J.-F. Cryst. Growth Des. 2016, 16, 4547-4558.

(19) Terban, M. W.; Cheung, E. Y.; Krolikowski, P.; Billinge, S. J. L. Cryst. Growth Des. 2016, 16, 210220.

(20) Jolliffe, I. T. 2002 Principal component analysis, 2nd edn. New York, NY: Springer-Verlag.

(21) Wang, Y.-X.; Zhang, Y.-J. IEEE Trans. Knowl. Data Eng. 2013, 25, 1336-1353.

(22) Chapman, K. W.; Lapidus, S. H.; Chupas, P. J. J. Appl. Cryst.

2015, 48, 1619-1626.

(23) Seung, H. S.; Lee, D. D. Nature 1999, 401, 788-791.

(24) Lee, D. D.; Seung, H. S. Adv. Neural Inf. Process Syst. 2001, 13, 556-562.

(25) Berry, M. W.; Browne, M.; Langville, A. N.; Pauca, V. P.; Plemmons, R. J. Comput. Stat. Data Anal. 2007, 52, 155-173.

(26) Soper, A. K. GudrunN and GudrunX: Programs for Correcting Raw Neutron and X-ray Diffraction Data to Differential Scattering Cross Section. Tech. Rep. RAL-TR-2011-013 (Rutherford Appleton Laboratory, 2011).

(27) Soper, A. K.; Barney, J. Appl. Cryst. 2011, 44, 714-726.

(28) Keen, D. A.; J. Appl. Cryst. 2001, 34, 172-177.

(29) Marsac, P. J.; Konno, H.; Taylor, L. S. Pharm. Res. 2006, 23, 2306-2316.

(30) Strictly, $G_{3}^{*}(r)$ will comprise the PDF of amorphous felodipine together with contributions from interfaces between different phases (e.g. $\mathrm{API} /$ binder). We make use of the same approximation exploited in difference PDF measurements ${ }^{22,37}$ that inter-phase PDFs are substantially less structured than intra-phase PDFs and hence $G_{3}^{*}(r)$ will be dominated by the contribution from amorphous API.

(31) Zidan, A. S.; Rahman, Z.; Sayeed, V.; Raw, A.; Yu, L.; Khan, M. A. Int. J. Pharm. 2012, 423, 341-350.

(32) Rumondor, A. C. F.; Taylor, L. S. Int. J. Pharm. 2010, 398, 155-160.

(33) Vo, C. L.-N.; Park, C.; Lee, B.-J. Eur. J. Pharm. Biopharm. 2013, 85, 799-813.

(34) Baird, J. A.; Taylor, L. S. Adv. Drug Deliv. Rev. 2012, 64, 396-421.

(35) Langham, Z. A.; Booth, J.; Hughes, L. P.; Reynolds, G. K.; Wren, S. A. C. J. Pharm. Sci. 2012, $101,2798-2810$

(36) Fossheim, R. J. Med. Chem. 1986, 29, 305307.

(37) Chupas, P. J.; Chapman, K. W.; Jennings, G.; Lee, P. L.; Grey, C. P. J. Am. Chem. Soc. 2007, 129, 13822-13824. 
